\title{
Characterizing Turbulent Ceiling Jet Dynamics with Salt-water Modeling
}

\author{
XIAOBO YAO and ANDRÉ W. MARSHALL \\ Department of Fire Protection Engineering \\ University of Maryland, College Park \\ University of Maryland \\ 0151 Martin Hall \\ College Park, Maryland 20742
}

\begin{abstract}
Ceiling jet dynamics are quantitatively characterized using the salt-water modeling technique. In this study, salt-water is introduced into fresh water to create an axisymmetric impinging plume and the associated stratified wall jet. This configuration models the canonical ceiling jet flow that results from impinging fire plumes. The saltwater modeling analogy is demonstrated through rigorous scaling of the governing equations and comparison of the resulting fire and salt-water source-based dimensionless quantities. With appropriate scaling, the measured concentrations in the salt-water model can be directly compared with temperatures in the fire configuration. Measurements of the mixing and dispersion in the ceiling jet region are performed using Planar Laser Induced Fluorescence (PLIF). These measurements provide quantitative visualization of the turbulent flow structure in addition to mean concentration profiles. Ceiling layer thicknesses, peak profile values, and profile shapes along the ceiling compare favorably with ceiling jet theory and data from actual fire experiments. Furthermore, turbulent flow details are revealed through the laser sheet visualization providing insight into the flow behavior and providing quantitative data for CFD code validation.
\end{abstract}

KEYWORDS: ceiling jet, turbulence, PLIF, salt-water, heat transfer

\section{NOMENCLATURE}

$Y_{S}$ salt mass fraction

$H \quad$ 'ceiling' height above source

$l_{T} \quad$ ceiling layer thermal thickness

$L_{M} \quad$ Morton scale (m), describes momentum to buoyancy ratio

$V \quad$ salt-water injection flow rate

$\dot{Q}$ energy release rate

$U$ ceiling jet velocity

$h \quad$ ceiling jet thickness

$t^{*} \quad$ dimensionless time, $=t(g / H)^{1 / 2}\left(\chi^{*}\right)^{1 / 3}$

$D^{*} \quad$ plume length scale, $=\left(\chi^{*}\right)^{2 / 5} x_{3}$

$x_{i} \quad$ position vector $(\mathrm{m})$

$x_{i}^{*} \quad$ dimensionless position, $=x_{i} / H$

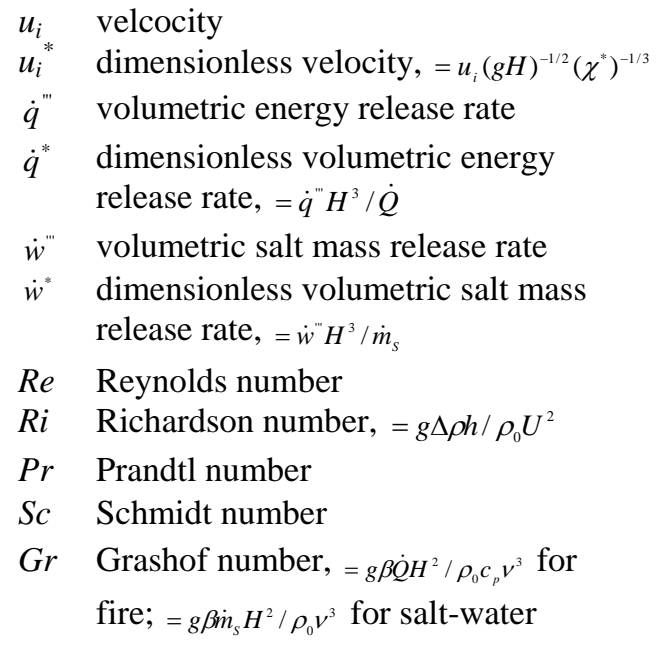




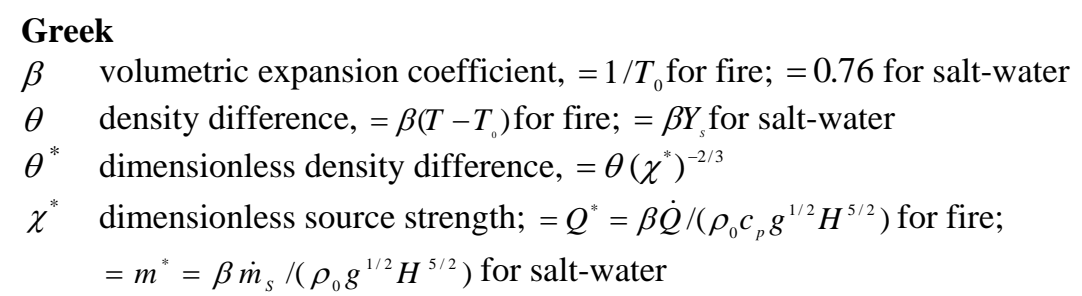

\section{INTRODUCTION}

The interaction of the fire plume with the ceiling plays a major role in critical physical processes of engineering relevance including smoke dispersion important in toxicity and detection and heat loading important in fire-structure interaction and flame spread. A number of theoretical and experimental fire studies have been performed in this flow configuration. Most notably, Alpert [1] performed an analytical and experimental study developing the theory and associated scaling laws for fire induced ceiling jets. Based on his analysis, he provided relationships for dimensionless ceiling layer thickness, velocity, and temperature, which compared favorably with measurements. In fact, his analysis revealed that these flow quantities are relatively insensitive to geometric scale. Alpert suggested that credible small-scale experiments could be conducted at ceiling heights down to $0.6 \mathrm{~m}$. At smaller scales, laminarization of the plume became an issue. Motevalli and Marks [2] conducted small-scale experiments of ceiling jet heat transfer, which generally compared favorably with other ceiling jet data and analysis [1-4] for $x_{1} / H<2$. However significant discrepancies were encountered when comparing measured momentum and thermal thickness between investigations. These discrepancies were attributed to coarse measurements by other investigators and simplifying assumptions concerning the equivalency of the momentum and thermal thicknesses in other analysis.

Salt-water modeling has been used to study fire induced flows in a variety of configurations. In salt-water modeling, a full-scale fire induced flow is simulated by creating turbulent buoyancy driven flow in a small-scale geometrically similar configuration. Steckler et al. [5] demonstrated this analogy through qualitative scale analysis and salt-water flow visualization experiments. In their study, they demonstrated that with appropriate scaling the governing equation for dimensionless temperature and dimensionless salt-water concentration has identical form with the exception of $R e, S c$, and $\operatorname{Pr}$ discrepancies encountered in diffusive term coefficients. They asserted that for sufficiently turbulent salt-water and fire-induced flow, diffusive discrepancies could be neglected owing to the small value of the diffusive term coefficients, thus establishing the analogy. However, it should be noted that very near the wall the diffusive terms and the corresponding discrepancies are not negligible. Kelly [6], using similar dye visualization techniques, a salinity probe, and scaling arguments, found semi-quantitative agreement when comparing dimensionless event times between salt-water experiments and computational fluid dynamics (CFD) analysis of fires in geometrically similar multi-room compartments. Clement and Fleischman [7] also compared salt-water experiments in a two-room compartment with CFD analysis using the Fire Dynamics Simulator (FDS). These salt-water experiments were conducted using Planar Laser Induced Fluorescence (PLIF) to obtain detailed salt concentration data. The LIF technique allows measurement of the salt-water concentration through tracking a fluorescent dye tracer, which is diluted at the same rate as the salt-water [8]. The salt-water concentration was used to determine the density field, which compared favorably with FDS predictions of density distributions in the plume and in the farfield adjacent to the source room. More recently, Jankiewicz 
[9] also combined salt-water modeling and PLIF to explore the applicability of these techniques to the prediction of detector response times. He found excellent agreement between dimensionless front arrival times in the salt-water model and the full-scale fire experiments. He also found some limited agreement between the dimensionless temperatures measured in the fire tests and dimensionless concentrations measured in the salt-water models. More impressive quantitative agreement between PLIF salt-water measurements and fire plume measurements has been demonstrated by Yao et al. [10,11] in the unconfined plume configuration. Yao compared his scaled salt-water measurements with McCaffrey's fire plume centerline temperature measurements [12] and point source plume theory. This comparison is provided in Fig. 1. The favorable agreement between PLIF salt-water measurements, fire plume measurements, and point source plume theory establishes the applicability of the PLIF salt-water measurement and scaling techniques to unconfined fire plumes. Yao et al. also obtained highly resolved measurements for sub-grid scale CFD model validation in that study.

In the current investigation, the salt-water modeling technique is applied to another canonical fire induced flow. The impinging plume and resulting ceiling jet are critically examined in this study. Salt-water measurements in this flow configuration are compared with fire measurements and ceiling jet theory. An illustration of this flow configuration is provided in Fig. 2. This ceiling jet study provides a test of the salt-water analogy in a simple geometry where wall effects can be important. Furthermore, the detailed measurements taken in this configuration provide much needed near-wall stratified flow data for CFD wall-model development and validation.

\section{METHODOLOGY}

Detailed quantitative salt-water modeling of fire induced flows requires the development of sophisticated experiments and careful scale analysis. An experimental facility was used in this experiment to carefully control and measure salt-water injection. Laser diagnostics were also employed to measure the mean and turbulent dispersion characteristics of the impinging plume and ceiling jet. Detailed scale analysis was also conducted in order to determine the appropriate dimensionless quantities for use in saltwater and fire induced flow configurations.

\section{Experimental Facility}

Salt-water experiments were conducted by gently introducing salt-water into quiescent fresh water through a small tube directed vertically downward toward a horizontal flat plate or 'ceiling'. This configuration simulates an impinging fire plume and the resulting ceiling jet. It should be noted that the salt-water flow is inverted; however, the integrity of the analogy does not depend on the direction of the buoyancy force. An illustration of the experimental facility is provided in Fig. 3. A constant gravity head tank is used to introduce the salt-water dye mixture. A $500 \mathrm{~mW}$ Argon-Ion laser is used with a cylindrical lens to create a $0.5 \mathrm{~mm}$ thick laser sheet to image the turbulent mixing in an $x_{1}-x_{3}$ plane of the impinging plume. Flow images are captured using a digital SLR camera fitted with a normal lens, a high pass interference filter, and image processing software. After the flow has developed, 500 images are acquired at about $1 \mathrm{~Hz}$ with exposure times of $1 / 60 \mathrm{~s}$. 


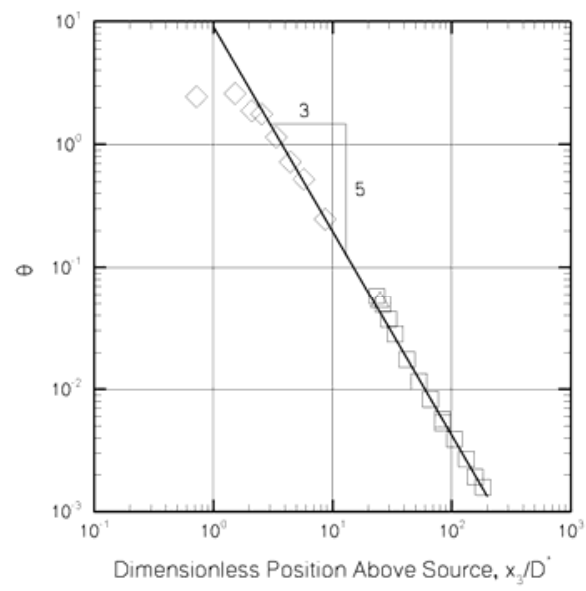

Fig. 1. Centerline mean density difference; $\diamond$ McCaffrey's plume; salt-water model $\square V=150 \mathrm{ml} / \mathrm{min} ; \triangle V=110 \mathrm{ml} / \mathrm{min}$; — theory.

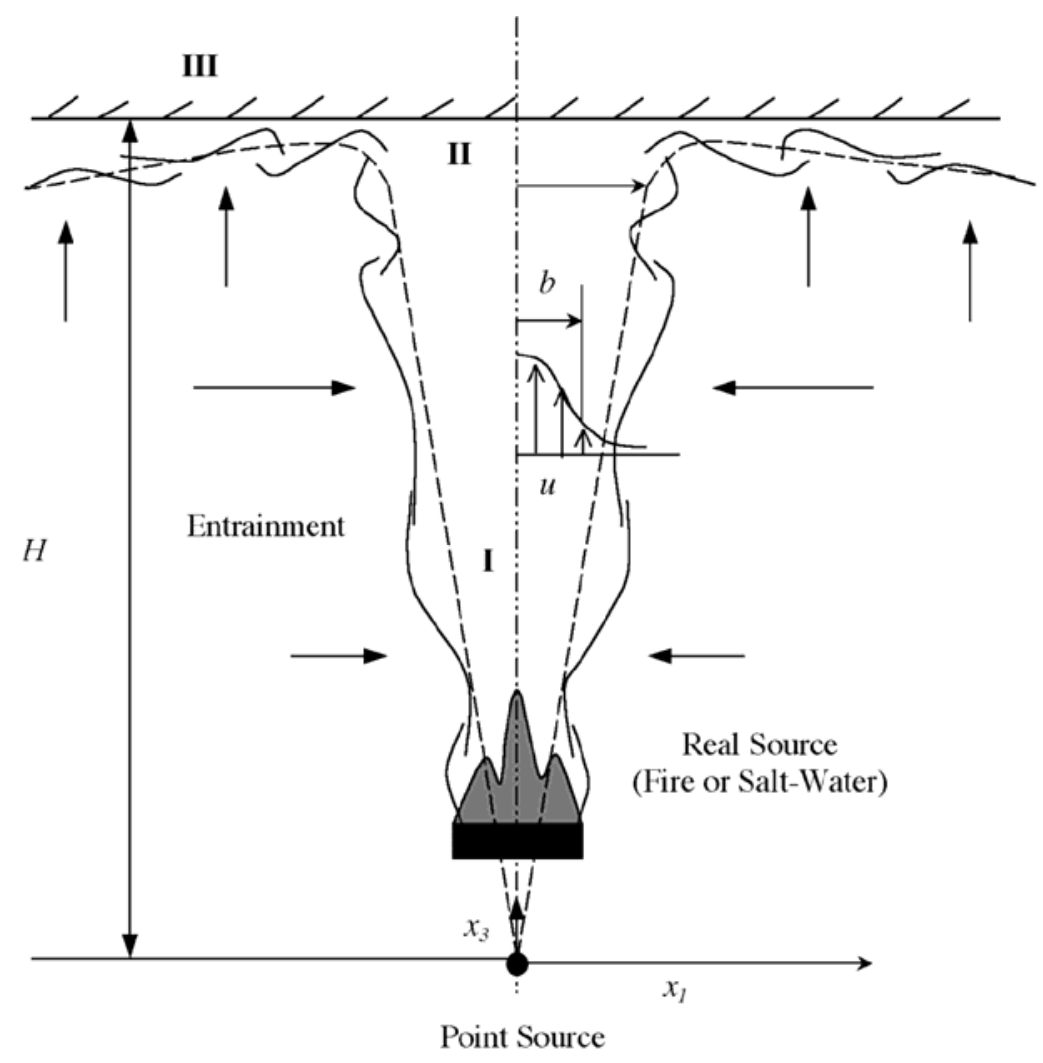

Fig. 2. Impinging plume and ceiling jet configuration; I. plume region; II. impingement/turning region, III. ceiling jet region. 


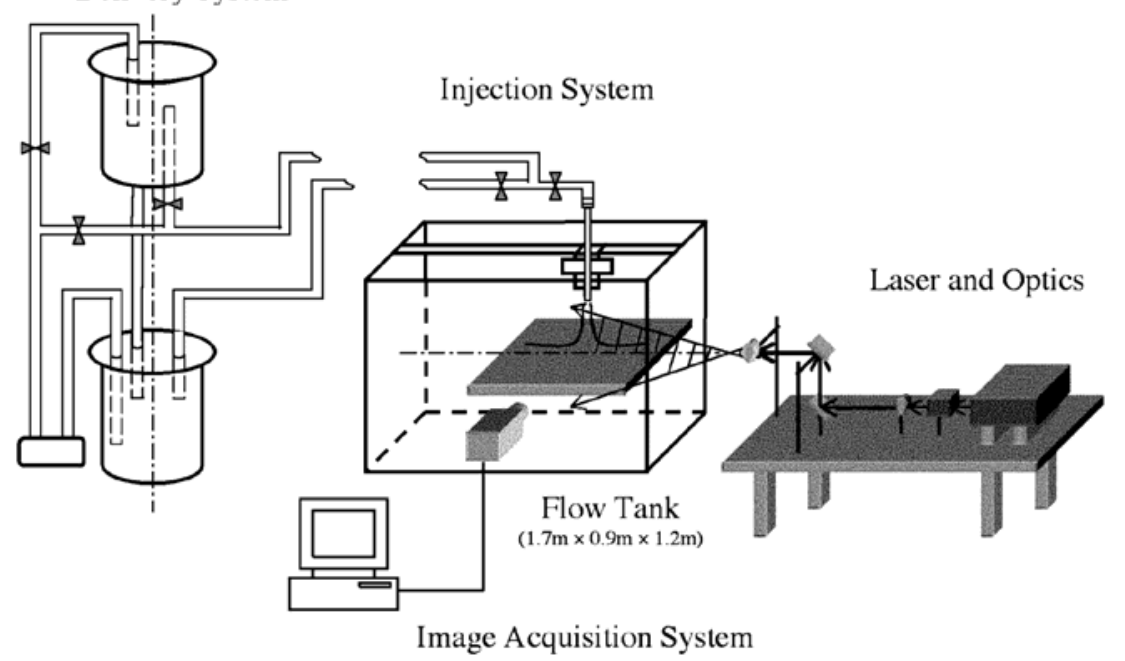

Fig. 3. Quantitative salt-water modeling facility.

\section{Laser Diagnostics}

Quantitative flow visualization is performed in this study using Planar Laser Induced Fluorescence (PLIF). In these PLIF experiments, a fluorescent dye tracer of known concentration is mixed with the injected salt-water. This dye salt-water solution advances downward toward a horizontal 'ceiling' while mixing with the surrounding fresh water. A laser sheet having a wavelength capable of exciting the fluorescent dye is used to illuminate a region of interest within the flow. The light fluoresced from the dye, having its own distinct wavelength, produces an image that is collected with a camera placed at a right angle to the light sheet. The intensity of the fluorescent light is directly proportional to the dye concentration and the corresponding dispersed salt concentration. The salt mass fraction is easily determined from the measured concentrations. However, it should be noted that numerous experimental and image processing procedures are necessary to obtain quantitative data from the raw intensity images. These procedures include calibration for the relationship between fluorescence intensity and salt concentration, prevention of fluorescence absorption, removal of background noise, and correction for the incident light sheet distribution. These procedures and their ability to produce credible quantitative data are discussed in detail in Jankewicz [9] and Yao [10,11]. If data near-solid boundaries are of interest, care must be taken to minimize wall reflections. Through introduction of the laser sheet along a 'ceiling' with a matted surface, wall reflection effects were isolated to within $0.004 H$ of the 'ceiling'. Although implementation of the PLIF technique is rather involved, it has the advantage of allowing for observation of the flow structures responsible for mixing and dispersion while providing quantitative salt mass fractions. These salt mass fractions are useful for fire induced dispersion analysis (w/ appropriate scaling) and analysis of buoyant turbulent flow details. 


\section{Experimental Conditions}

For this study, an injector diameter of $D=5.6 \mathrm{~mm}$, injection salt-water flow rate of $V=$ $110 \mathrm{ml} / \mathrm{min}$, injection salt mass fractions of $\left(Y_{S}\right)_{i n j}=0.13$, and Rhodamine $6 \mathrm{G}$ dye concentrations ranging from $0.2 \mathrm{mg} / \mathrm{l}<[D Y E]<0.5 \mathrm{mg} / \mathrm{l}$ were used. The injector diameter, injection flow rate, and salt mass fraction are designed to facilitate a quick transition from momentum dominated jet behavior to buoyancy dominated plume behavior. Morton [13] proposed a length scale, $L_{M}$, for buoyant jets to transition to plume-like behavior. Morton suggested that plume-like behavior will be achieved at $5 L_{M}$ downstream of the point of injection. For the experiments in this study, $5 L_{M}$ is less than $0.2 H$, where $H$ is the ceiling jet height. The small plume development length ensures that the impinging plume and resulting ceiling jet will be governed by buoyant plume dynamics. Similarly, ceiling jet theory and correlations proposed by Alpert [1] and others require that the fire be small compared with ceiling height to ensure that the flow is governed by buoyant plume dynamics.

\section{Scaling}

In order to compare salt-water and fire induced flows, it is important to establish the analogous dimensionless parameters. These parameters become apparent when comparing the energy equation for fire induced flows and the salt mass transport equation for salt-water flows. These equations were developed in detail in Jankiewicz [9].

The energy equation for the fire configuration is given by

$$
\frac{\partial \theta^{*}}{\partial t^{*}}+u_{i}^{*} \frac{\partial \theta^{*}}{\partial x_{i}^{*}}=\frac{1}{G r^{1 / 3} \operatorname{Pr}} \frac{\partial^{2} \theta^{*}}{\partial x_{i}^{*} \partial x_{i}^{*}}+\dot{q}^{*}
$$

where $\theta^{*}$ is the source based dimensionless temperature rise, $\theta^{*}=\beta\left(T-T_{o}\right)\left(Q^{*}\right)^{-2 / 3}$, which is equivalent to the source based dimensionless density difference. The dimensionless variables and parameters are based on the dimensionless source strength, which is $Q^{*}=\beta \dot{Q} / \rho_{o} c_{p} g^{1 / 2} H^{5 / 2}$ in the fire induced flow configuration and $m^{*}=\beta \dot{m}_{s} / \rho_{o} g^{1 / 2} H^{5 / 2}$ in the salt-water configuration. It should be noted that for a point source, $\dot{q}^{*}=0$ everywhere except at the source. In the salt-water flow, the transport equation for the salt mass fraction is given by

$$
\frac{\partial \theta^{*}}{\partial t^{*}}+u_{i}^{*} \frac{\partial \theta^{*}}{\partial x_{i}^{*}}=\frac{1}{G r^{1 / 3} S c} \frac{\partial^{2} \theta^{*}}{\partial x_{i}^{*} \partial x_{i}^{*}}+\dot{w}^{*}
$$

where $\theta^{*}$ is the source based dimensionless mass fraction, $\theta^{*}=\beta Y_{s}\left(m^{*}\right)^{-2 / 3}$, which is equivalent to the source based dimensionless density difference. In the dimensionless salt transport equations, similarly $\dot{w}^{*}=0$ everywhere except at the source. For unconfined flows, such as the free plume, details regarding the boundaries in the fire and salt-water configurations need not be considered. However, if ceilings or walls are present in the flow domain of interest, the boundary condition in the salt-water configuration requires some interpretation. The impermeable boundary condition resulting in zero mass fraction 
gradients at the wall in the salt-water configuration is analogous to the adiabatic boundary condition in the fire configuration. At this point, it should be noted that the equations and boundary conditions governing dimensionless density difference in the fire induced flow configuration and salt-water configuration have similar form. The dimensionless density difference is thus expected to have similar behavior if the dimensionless parameters are matched. However, as discussed previously, discrepancies in the $R e=G r^{1 / 3}, P r$, and $S c$ between the fire and salt-water configurations may cause the flows to behave differently. Nevertheless, if the $R e$ is sufficiently large and the flow is turbulent, molecular diffusion will be small and differences in molecular properties and their associated dimensionless parameters can be neglected.

General guidelines pertaining to $R e$ requirements have not yet been established for saltwater modeling of fire induced flows. Furthermore, the associated potential importance of wall effects remains largely unexplored in these salt-water modeling applications. This study represents a first step in quantitatively comparing salt-water and fire induced flows in a simple configuration where both $R e$ and wall effects may be important. The simplicity of the impinging plume configuration, the detailed measurements provided by PLIF, and the theoretical analysis already developed for this configuration make it ideal for detailed comparisons of salt-water and fire induced flows.

\section{RESULTS AND DISCUSSION}

Detailed salt dispersion measurements in the salt-water configuration are carefully compared to temperature measurements and models from a variety of investigators. The salt-water experiments reveal the turbulent flow behavior of the impinging plume and ceiling jet, which is useful for detailed CFD model validation. The salt-water modeling results also provide mean measurements for comparison with the analytical treatment of this problem and actual fire data.

The gross flow features of the impinging plume and resulting ceiling jet are easily observed in the average and standard deviation images of salt dispersion shown in Fig. 4a. Recall that the dimensionless density difference provides a direct measure of the salt mass fraction in the salt-water configuration and the temperature rise in the fire induced flow configuration. The decay in the dimensionless density difference is clearly observed in the plume region as the flow approaches the ceiling. The spreading of the plume in the turning region is clearly observed resulting in a slender radial expanding ceiling jet. The qualitative behavior of the flow is consistent with expectations. Furthermore the instantaneous impinging plume shown in Fig. 4b provides a substantially different view of the flow when compared with the corresponding mean image. Peak values of the dimensionless density difference occur in the ceiling layer near the wall, but not at the wall as has been suggested by other investigators [3]. The turbulent structure of the salt-water flow and its interaction with the wall is clearly evident in this instantaneous impinging plume image even at the relatively low Grashof number, $G r=1.1 \times 10^{11}$, of this small-scale salt-water flow. In certain regions, ambient fluid appears to penetrate all the way to the wall. 
$\theta^{*}$

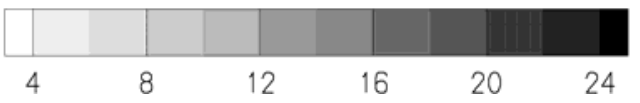

$\theta^{*}$ given by (colormap value) $\times(0.4)$ for standard deviation image

(a)

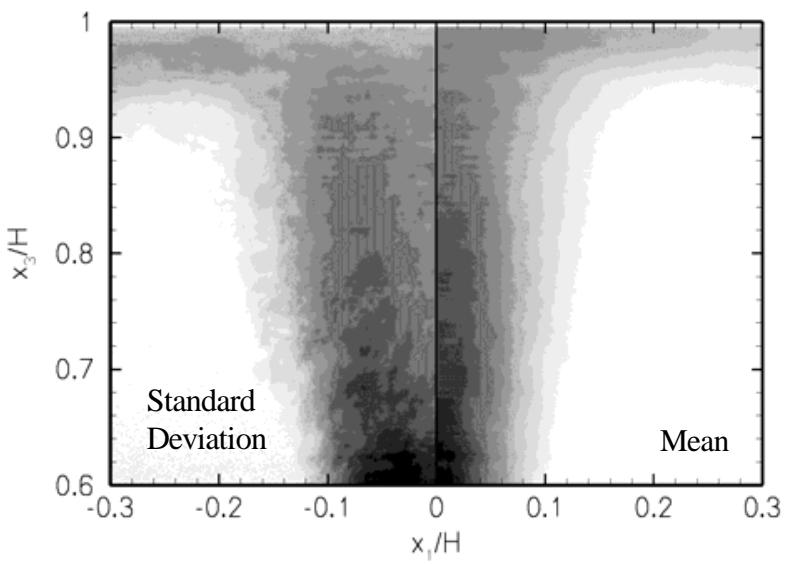

(b)

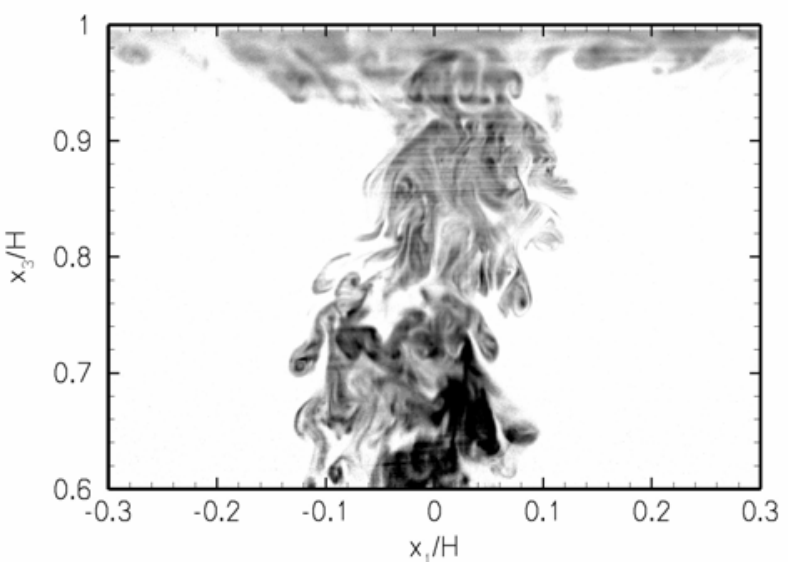

Fig. 4. Quantitative visualization of the dimensionless density difference in salt-water flow, $\theta^{*}$; (a) standard deviation image, left and mean image, right; (b) instantaneous image.

Observation of instantaneous images at different locations along the ceiling reveal a clear trend in the evolution of turbulent structures that govern flow and thermal transport in the ceiling jet. Representative instantaneous images are provided at select positions along the ceiling in Fig. 5. Upstream of the impingement region, fresh fluid is entrained deep within the plume creating large-scale unmixed vertical structures characterized by sharp density interfaces. However, very near the ceiling, the sharp interfaces between the freshly entrained flow and the plume flow disappear even within the impingement region. The steep gradients introduced by the wall result in smaller length scales and associated smaller turbulent length scales, which act to remove these sharp interfaces. The ceiling jet emerges from the impingement region, still turbulent, with a non-uniform but relatively smooth variation in density difference. Figure 5b shows typical ceiling jet behavior downstream of the impingement region. It should be noted that the images in Figs. 5b and 


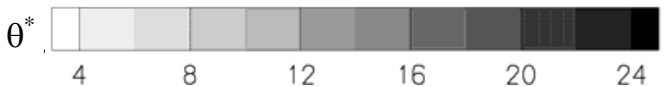

$\theta^{*}$ given by (colormap value) / (4) for images (b) and (c)

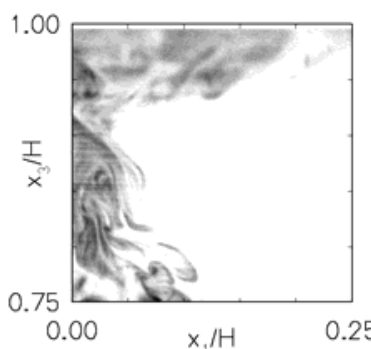

(a)

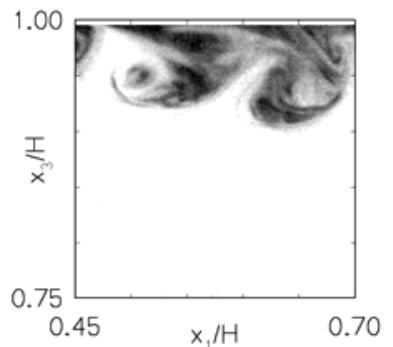

(b)

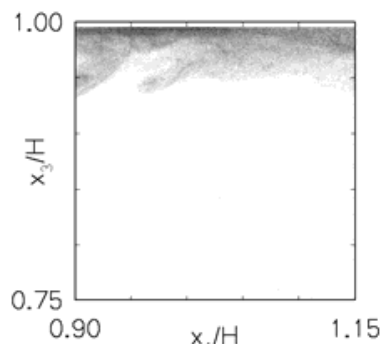

(c)

Fig. 5. Turbulent structures and ceiling jet progression; (a) impingement and turning region $0<x_{1} / H<0.25$; (b) ceiling jet region $0.45<x_{1} / H<0.7$;

(c) ceiling jet region $0.9<x_{1} / H<1.15$.

5c have been 'brightened' by a factor of 4 to improve contrast. Color map values must be divided by 4 to obtain actual $\theta^{*}$ in these regions. Large scale turbulent motion is still observed along the ceiling jet occasionally penetrating to the wall indicating that entrainment is important. It is well established that the entrainment will be reduced along the ceiling layer as the Richardson number, $R i=g \Delta \rho h / \rho_{0} U^{2}$ increases [1]. The $R i$ describes the ratio of potential energy associated with density differences (which inhibits mixing and entrainment) and kinetic flow energy (which promotes mixing and entrainment). Further away from the impingement region, very little turbulence is observed, as shown in Fig. 5c. Occasional turbulent structures are observed on the surface of the layer, but never penetrate to the wall. In this region, the $R i$ has increased to the extent that entrainment is almost completely inhibited. Determination of the exact value of the local $R i$ will be possible through velocity measurements planned in future work.

Salt-water flows are directly and quantitatively compared to fire induced flows through the dimensionless density difference decay along the ceiling corresponding to peak salt mass fraction and temperature rise, respectively. Figure 6a shows the dimensionless density difference from the salt-water flow configuration, small-scale experiments conducted by Motevalli [2] and Veldman [3], correlations developed by Cooper [14], and analysis conducted by Alpert for large scale fires [1]. At $x_{1} / H<0.5$, the quantitative agreement between similarly scaled salt-water and fire measurement is quite impressive. The smaller $G r$ in the salt-water configuration makes the flow more sensitive to $R i$ effects. As mentioned previously, $R i$ increases along the ceiling result in reduced entrainment as buoyancy acts to stably stratify the ceiling layer, inhibiting mixing. In a low $\mathrm{Gr}$ flow, this action will not only result in reduced entrainment, but also in laminarization of the ceiling layer. This effect is clearly observed along the ceiling in the region $x_{1} / H>0.5$. In this region, the salt-water dimensionless density difference decay along the ceiling begins to deviate sharply from the data provided from the fire induced flow configuration. The thermal thickness of the ceiling layer, $l_{T}$, was also determined. This quantity provides an indication of the ceiling jet thickness. It is defined as the distance from the ceiling where $\theta^{*} / \theta_{\text {MAX }}^{*}=1 / e$. The thermal thickness data presented in Fig. 6b compares favorably with Motevalli correlation up to $x_{1} / H=0.6$. Beyond this 
point, the flow begins to laminarize profoundly reducing entrainment resulting in layer thinning consistent with a radially expanding stratified layer. Alpert's theory and Cooper's correlations tend to under-predict the thermal thickness for $x_{1} / H<1.0$. In fact, Alpert [15] reports good agreement with Motevalli's thermal thickness correlation in later experiments.

To further evaluate ceiling jet transport, normalized ceiling layer density difference profiles are compared at various $x_{1} / H$ along the ceiling in Fig. 7 . The salt-water profile is described extremely well with a half-Gaussian profile, which is consistent with Alpert's recommendation. The near zero $\theta^{*}$ gradient achieved close to the ceiling is consistent with the adiabatic wall analogy used in salt-water modeling. Motevalli's correlation compares favorably with this Gaussian behavior as well, except near the ceiling where the effect of heat losses becomes evident through steep wall gradients and off-ceiling peak temperatures. These heat losses persist despite efforts taken to achieve an adiabatic wall in the fire configuration. The ability of the salt-water modeling to produce an ideal canonical flow configuration is clearly demonstrated through this comparison.

(a)

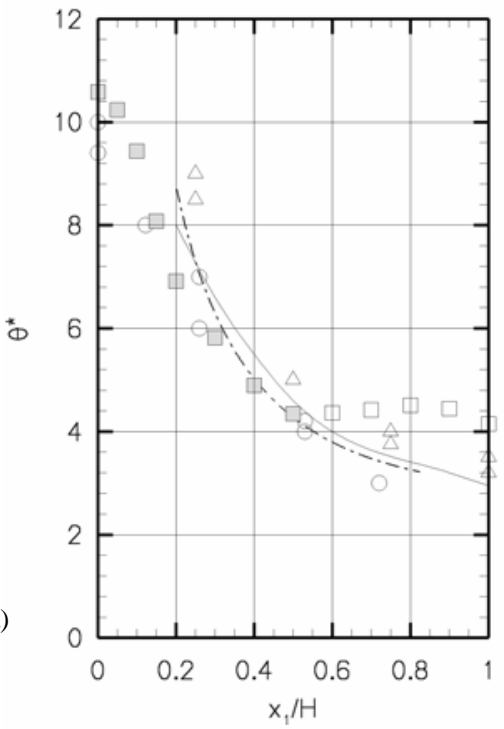

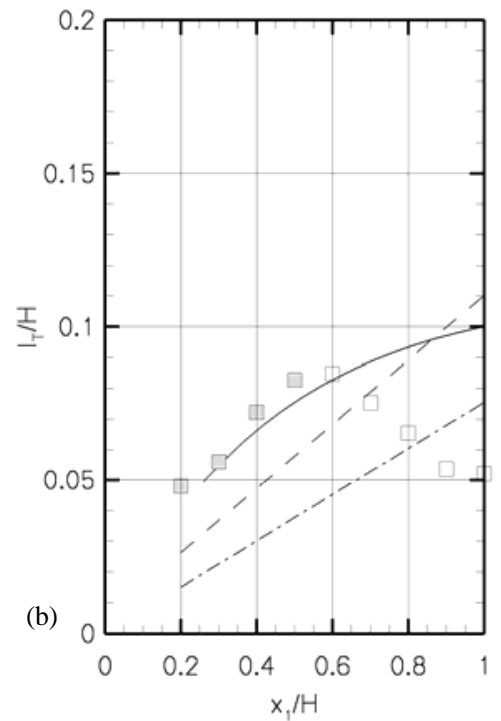

Fig 6. Comparison of dimensionless density difference magnitudes along the ceiling;

(a) peak values; (b) peak value locations; $\square$ salt-water model; $\square$ salt-water model after laminarization; $\triangle$ Motevalli [2]; $\bigcirc$ Veldman [3]; —— Alpert theory

[1]; - - - Cooper correlation [14]; - - - - Motevalli correlation [2]. 


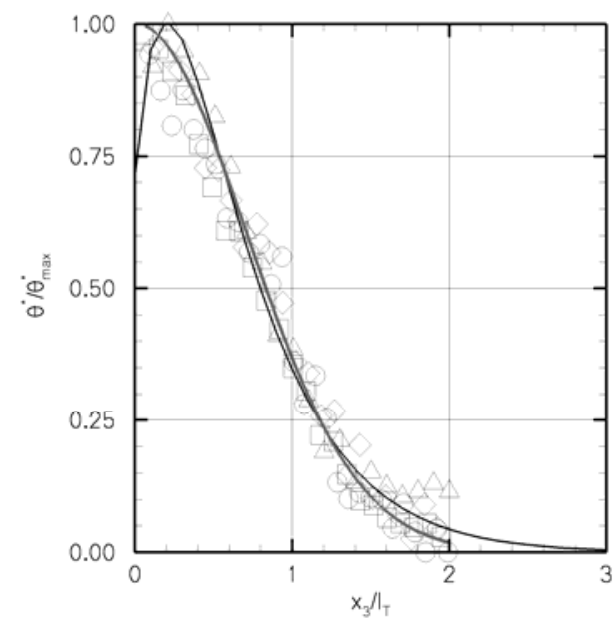

Fig. 7. Mean profiles of dimensionless density difference at various locations along the ceiling jet; $\triangle r / H=0.2 ; \diamond r / H=0.4 ; \bigcirc r / H=0.6 ; \square r / H=0.8$;

Gaussian curve fit, $1 / \exp \left(x_{3} / l_{T}\right)^{2} ;-$ Motevalli correlation [2].

\section{CONCLUSIONS}

Salt-water modeling has been used in many investigations to qualitatively study the behavior of fire induced flows. The low $R e$ and its impact on near wall behavior have long raised questions concerning the accuracy of salt-water modeling and its potential to be used as a quantitative method. In this study, PLIF measurements of salt dispersion were performed in a salt-water ceiling jet configuration (with ceiling height of $0.2 \mathrm{~m}$ corresponding to a $G r=1.1 \times 10^{11}$ ) for detailed comparison with fire induced ceiling jet measurements. Scaling the governing transport equations revealed that the dimensionless density difference, $\theta^{*}$, is the appropriate analogous quantity for comparison of these flows. The dimensionless density difference is related to the salt mass fraction in the saltwater model and the temperature rise in the fire induced flow configuration. The detailed salt-water modeling measurements in the canonical ceiling jet flow quantify the near wall behavior of salt-water flows and provide some guidance on when wall induced errors due to $R e$ discrepancies become unacceptable. For salt-water flow configurations, a $G r=1.1$ $\times 10^{11}(R e \approx 4800)$ provided excellent agreement with fire test data (within $\left.10 \%\right)$ at ceiling locations having $x_{1} / H<0.5$. However, for $x_{1} / H>0.5$, the over-prediction of dimensionless density difference in the salt-water is unacceptable. Flow visualization revealed that only weak turbulence persists in this region due to the relatively low $R e$ in salt-water modeling flows, which significantly reduces entrainment and prevents the expected continuous decay of the dimensionless density difference along the ceiling. Larger $G r$ would provide salt-water modeling fidelity beyond $x_{1} / H>0.5$. Alternatively, reducing the $\mathrm{Gr}$ below $1.1 \times 10^{11}$ is not advised for quantitative results. At lower Grashof numbers, $G r=5.5 \times 10^{10}$, the investigators found that the plume turbulence is not fully developed at the entrance to the impingement region resulting in anomalous behavior along the ceiling.

\section{ACKNOWLEDGEMENTS}

The authors would like to gratefully acknowledge Combustion Science and Engineering, Inc. (CSE) for their support of this research. 


\section{REFERENCES}

[1] Alpert, R.L., "Turbulent Ceiling-jet Induced by Large-scale Fires,” Combustion Science and Technology, 11, pp. 197-213, (1975).

[2] Motevalli, V., and Marks, C., "Characterizing the Unconfined Ceiling Jet under Steady-state Conditions: A Reassessment,” Fire Safety Science -- Proceedings of the Third International Symposium, International Association for Fire Safety Science, 1991, pp. 301-312.

[3] Veldman, C.C., Kubota, T., and Zukoski, E.E., “An Experimental Investigation of the Heat Transfer from a Buoyant Gas Plume to a Horizontal Ceiling - Part I: Unobstructed Ceiling,” NIST Rep. NBS-GCR-77-97, Gaithersburg, MD, 1975.

[4] You, H.Z., and Faeth, G.M., "An Investigation of Fire Impingement on a Horizontal Ceiling,” NIST Rep. NBS-GCR-79-188, Gaithersburg, MD, 1978.

[5] Steckler, K., Baum, H., and Quintiere, J., "Salt-water Modeling of Fire Induced Flows in Multicompartment Enclosures," Twenty-first Symposium (International) on Combustion, The Combustion Institute, 1986, pp. 143-149.

[6] Kelly, A.A., "Examination of Smoke Movement in a Two-story Compartment Using Salt-water and Computational Fluid Dynamics Modeling,” M.S. Thesis, University of Maryland, 2001.

[7] Clement, J.M., and Fleischmann, C.M., "Experimental Verification of the Fire Dynamics Simulator Hydrodynamic Model,” Fire Safety Science -- Proceedings of the Seventh International Symposium, International Association for Fire Safety Science, 2002, pp. 839-851.

[8] Walker, D.A., "A Fluorescence Technique for Measurement of Concentration in Mixing Liquids,” Journal of Physics E: Sci. Instrum, 20, pp. 217-224, (1987).

[9] Jankiewicz, S.P. "Prediction Smoke Detector Response Using A Quantitative Salt-water Modeling Technique,” M.S. Thesis, University of Maryland, 2001.

[10] Yao, X., and Marshall, A.W., "Quantitative Salt-water Modeling of Fire Induced Flow,” Fire Safety Journal, (2005), (submitted).

[11] Yao, X., Ma, T., Marshall, A.W., and Trouvé, A., "Mixing and Turbulent Transport in Unconfined and Impinging Plumes," $10^{\text {th }}$ International Fire Science and Engineering Conference - interflam, Edinburgh, Scotland, 2004.

[12] McCaffrey, B.J., "Purely Buoyant Diffusion Flames: Some Experimental Results,” NIST Rep. NBSIR 79-1910, Gaithersburg, MD, 1979.

[13] Morton, B.R., “Forced Plumes,” Journal of Fluid Mechanics, 5, pp. 151-163, (1959).

[14] Cooper, L.Y., "Fire-plume-generated Ceiling Jet Characteristics and Convective Heat Transfer to Ceiling and Wall Surfaces in a Two-layer Fire Environment: Uniform Temperature Ceiling and Walls,” Fire Science \& Technology, 13, pp. 1-17, (1993).

[15] Alpert, R. L., "Ceiling Jet Flows," The SFPE Handbook of Fire Protection Engineering ( ${ }^{\text {nd }} e d$ ), DiNenno P.J. (ed.), National Fire Protection Association, Quincy, MA, 2002, p. 2/18. 